\section{STRUCTURAL} BIOLOGY

ISSN 2059-7983

\title{
XModeScore: a novel method for accurate protonation/tautomer-state determination using quantum-mechanically driven macromolecular X-ray crystallographic refinement
}

\author{
Oleg Borbulevych, ${ }^{a}$ Roger I. Martin, ${ }^{a}$ Ian J. Tickle ${ }^{b}$ and Lance M. Westerhoff ${ }^{a *}$
}

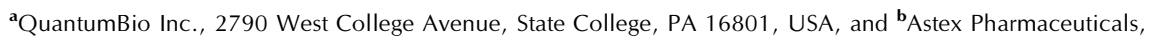
436 Science Park, Milton Road, Cambridge CB4 0QA, England. *Correspondence e-mail: lance@quantumbioinc.com

Edited by R. J. Read, University of Cambridge, England

Keywords: X-ray crystallography; quantummechanics X-ray refinement; ligand strain; highthroughput crystallography; protonation states; tautomers; difference density Z score; structureguided drug discovery; structure-based drug discovery; XModeScore.

Supporting information: this article has supporting information at journals.iucr.org/d

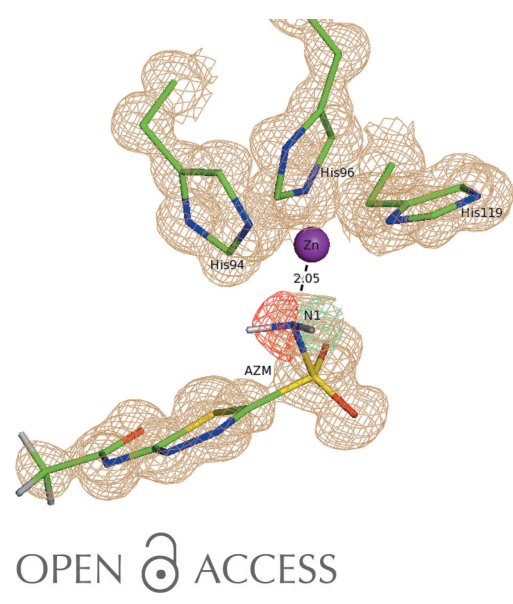

Gaining an understanding of the protein-ligand complex structure along with the proper protonation and explicit solvent effects can be important in obtaining meaningful results in structure-guided drug discovery and structure-based drug discovery. Unfortunately, protonation and tautomerism are difficult to establish with conventional methods because of difficulties in the experimental detection of $\mathrm{H}$ atoms owing to the well known limitations of $\mathrm{X}$-ray crystallography. In the present work, it is demonstrated that semiempirical, quantum-mechanics-based macromolecular crystallographic refinement is sensitive to the choice of a protonation-state/tautomer form of ligands and residues, and can therefore be used to explore potential states. A novel scoring method, called XModeScore, is described which enumerates the possible protomeric/tautomeric modes, refines each mode against $\mathrm{X}$-ray diffraction data with the semiempirical quantummechanics (PM6) Hamiltonian and scores each mode using a combination of energetic strain (or ligand strain) and rigorous statistical analysis of the difference electron-density distribution. It is shown that using XModeScore it is possible to consistently distinguish the correct bound protomeric/tautomeric modes based on routine X-ray data, even at lower resolutions of around $3 \AA$. These X-ray results are compared with the results obtained from much more expensive and laborious neutron diffraction studies for three different examples: tautomerism in the acetazolamide ligand of human carbonic anhydrase II (PDB entries $3 \mathrm{hs} 4$ and $4 \mathrm{k} 0 \mathrm{~s}$ ), tautomerism in the $8 \mathrm{HX}$ ligand of urate oxidase (PDB entries $4 \mathrm{n} 9 \mathrm{~s}$ and $4 \mathrm{n} 9 \mathrm{~m}$ ) and the protonation states of the catalytic aspartic acid found within the active site of an aspartic protease (PDB entry 2jjj). In each case, XModeScore applied to the X-ray diffraction data is able to determine the correct protonation state as defined by the neutron diffraction data. The impact of QM-based refinement versus conventional refinement on XModeScore is also discussed.

\section{Introduction}

Within structure-guided drug discovery (SGDD) and structurebased drug discovery (SBDD), accurate understanding of the protein-ligand complex structure, including the relevant proper protonation, is significant for obtaining meaningful results from docking/scoring, thermodynamic calculations, active-site exploration, lead optimization and, ultimately, medicinal chemistry (Pospisil et al., 2003). The most ubiquitous element in the universe is hydrogen, and these protons are critical for exploring the chemistry within the active site. For example, in the drug Mirapex, which is used to treat the symptoms of Parkinson's disease, the important chemical activity is conferred by a single aminothiazole tautomeric state rather than an alternative imino tautomer (Varga et al., 2009); thus the selection of the wrong state during drug design would lead to irrelevant findings. This situation is not uncommon, 
and drug discovery frequently hinges on the determination of one state versus another (Martin, 2010).

The most common method for structure determination in SGDD/SBDD is macromolecular X-ray crystallography. Unfortunately, an intrinsic problem of X-ray crystallography is its inability to explicitly detect $\mathrm{H}$ atoms, even at resolutions close to atomic, because the $\mathrm{H}$ atom has the weakest scattering power for X-rays among all elements (Rupp, 2009). H atoms are small, and their electrons are shifted towards the heavy atoms to which they are bound. Hence, it is generally extremely difficult to experimentally determine the protonation or tautomeric state of both the ligand and the surrounding active site. Protonation states can be unambiguously established using neutron diffraction because the neutron scattering length of deuterium is similar to that of heavy atoms (Bacon, 1975). Thus, the scattering by hydrogen/deuterium is comparable to that by other atoms in macromolecular structures. However, the prime disadvantage of this method that seriously limits its practical application is the considerable weakness of the neutron beam, leading to reliance on very large crystals and long exposure times for the collection of data of sufficient precision. Furthermore, $\mathrm{H}$ atoms have a negative scattering length, in contrast to the isotope deuterium (D); thus, the presence of $\mathrm{H}$ atoms gives rise to a cancellation during refinement against neutron data, reducing the sensitivity of the method (Afonine et al., 2010). Hence, complete deuteration of the sample crystal is highly desirable in a neutron diffraction experiment, but is practically difficult to achieve, since exchangeable protons constitute only about one quarter of all $\mathrm{H}$ atoms (Shu et al., 2000). Overall, neutron diffraction is rarely feasible within industrial SBDD settings.

In addition to the experimental limitations of X-ray crystallography with respect to proton detection, the stereochemical restraints used in conventional refinement are generally rudimentary in nature and do not account for interactions such as hydrogen bonds, dispersion, electrostatics, polarization and charge transfer (Adams et al., 2010; Kleywegt, 2007; Kleywegt et al., 2003). The DivCon linear scaling, quantum-mechanics (QM), semiempirical quantummechanics (SE-QM) and molecular-mechanics (MM) toolkit (Dixon \& Merz, 1996, 1997; QuantumBio Inc., http:// www.quantumbioinc.com) has been shown to capture the interactions between a target and its ligand(s) (Diller et al., 2010; Raha et al., 2005, 2007; van der Vaart \& Merz, 1999; Zhang et al., 2010). To address the deficiencies in conventional macromolecular refinement, we previously integrated DivCon with the PHENIX crystallographic package (Adams et al., 2010) to replace the conventional stereochemical restraint method with a more accurate quantum-based energy functional in 'real time' during refinement (Borbulevych et al., 2014; QuantumBio Inc.). Traditionally, we think of X-ray refinement as a balancing act between two components: the energy functional and the experimental density. However, with a more accurate (e.g. more trustworthy) functional, we can consider the input model, and its complement of atoms, as a third component. The success, as measured by agreement between the final model and experimental density, of an X-ray refinement campaign therefore depends on accuracy in all three components, and if upon completion of refinement there is disagreement between the model and the experimental density, this disagreement could be attributed to deficiencies in any of the components. For example, the functional could be missing a key interaction exhibited in the structure, there could be artifacts in the experiment, or the input model could be in an incorrect protonation state, thereby producing incorrect geometry. With PHENIX/DivCon, we are able to more accurately capture the key interactions within a proteinligand complex, including hydrogen bonds, dispersion, electrostatics, polarization, charge transfer, metal coordination etc. At the same time, crystallographers with greater proficiency and automation power are able to obtain better experimental densities. However, the question remains: can one create an approach or method that allows one to conclusively show which protonation state is most prevalent within the natural, biological structure?

To answer this question, we consider the fact that even though the $\mathrm{H}$ atom does not effectively scatter X-rays, with a more accurate functional we can observe the effects of these protons on the surrounding heavy atoms to determine whether or not the input protonation model is correct. By way of analogy, in 1845, John Couch Adams mathematically predicted the existence of the planet Neptune before its direct observation was made based upon unexplained perturbations in the orbit of the neighboring planet Uranus (Sampson, 1904). With experimental X-ray methods, we cannot directly observe $\mathrm{H}$ atoms; however, using the $\mathrm{QM} / \mathrm{MM}$ functional we are able to observe the influence of $\mathrm{H}$ atoms on the heavy atoms (carbon, nitrogen, oxygen) to which they are bound. Movements of such heavy atoms during QM/MM refinement results in certain changes in the electron-density maps, such as increasing/decreasing difference density peaks and correlation coefficients. The protomer/tautomer that produces the best fit to the experimental data can then be chosen based on statistical treatment (Tickle, 2012) of the results of the refinement of the structures with all possible protonation states.

With this hypothesis in mind, we developed the XModeScore technique, which couples the more advanced QM/MM methods in our refinement tool with a statistical comparison of the final model versus experimental density in order to determine whether or not the model is reflective of the actual chemistry within the structure. As opposed to score functions used in other fields, such as the affinity prediction functions used in docking/scoring algorithms, XModeScore 'scores' the various protonation modes using $\mathrm{X}$-ray density.

\section{Methods}

2.1. Validation method and structure selection, preparation and refinement

Neutron diffraction does not suffer from the same deficiencies as X-ray diffraction with regard to proton scattering, suggesting that these models can serve as 'gold standards' with which X-ray results can be compared. Further, since the 
XModeScore method is directly dependent upon the crystal, the method is sensitive to the actual protonation state found within that crystal, and therefore experimental conditions are important considerations. With this in mind, in order to choose a validation set we focused on those structures which (i) have both an X-ray diffraction model and a neutron diffraction model, (ii) have crystallization conditions (e.g. $\mathrm{pH}$, solvent, temperature etc.) which are approximately identical between the X-ray experiment and the neutron experiment, (iii) are complexed with chemically relevant or pharmaceutically interesting ligands and (iv) include deposited structure factors. The neutron diffraction model also had to be of a high enough quality that the key protonation states could be determined. Therefore, from the 88 neutron diffraction structures available in the PDB at the time of writing, the following three models were chosen.

(i) The AZM-human carbonic anhydrase II (HCA II) complex: neutron, PDB entry 4g0c; X-ray, PDB entry 3hs4 (Fisher et al., 2012).

(ii) The 8HX-urate oxidase complex: neutron, PDB entry 4n9m; X-ray, PDB entry 4n9s (Oksanen et al., 2014).

(iii) The PD-135,040-aspartic proteinase complex: neutron, PDB entry 2vs2; X-ray, PDB entry 2jjj (Coates et al., 2008). A second HCA II X-ray model, PDB entry 4k0s (A. Biswas, D. West, M. Pinard \& R. McKenna, unpublished work), was also selected in order to demonstrate the impact of resolution on $X M o d e S c o r e$ results.

The X-ray structures, along with their structure factors, were downloaded from the Protein Data Bank (PDB). H atoms were added to protein residues, water molecules and ligands using Protonate $3 D$ (Labute, 2009) as implemented in MOE2013 from Chemical Computing Group. Likely protomer/tautomer states were automatically generated with MOE2013 using the WashMoleculeMOE Scientific Vector Language (SVL) function. Since Protonate $3 D$ settles on a single protomeric/tautomeric state, after execution of WashMoleculeMOE each candidate protomeric/tautomeric state was fixed and Protonate $3 D$ was re-executed on the structure in order to 'propagate' proton addition/subtraction (along with corresponding residue rotameric flips) based upon each tautomer or protomer. In this way, protons are added/changed within the active site to match or counterbalance hydrogenbond changes within the ligand. QM region refinement as detailed previously (Borbulevych et al., 2011, 2014) was conducted on each structure using libQB (DivCon build-2577) incorporated into the PHENIX package v.1.9-1692 (Adams $e t$ al., 2010). The PM6 semiempirical QM Hamiltonian (Stewart, 2009; К̌ezáč et al., 2009) was used for each QM region, where each QM region was centered around the AZM ligand in PDB entries $3 \mathrm{hs} 4$ and $4 \mathrm{k} 0 \mathrm{~s}$, the $8 \mathrm{HX}$ ligand in PDB entry $4 \mathrm{n} 9 \mathrm{~s}$ and the key catalytic residue Asp215 in PDB entry 2jjj. For the region refinement, the default radii of 3.0 and $2.5 \AA$ for the main and buffer regions, respectively, were used. To explore the impact of resolution on each refinement and score, each structure was refined at several levels of data-set truncation using the phenix.refine keyword ' $x r a y \_d a t a . h i g h \_r e s o l u t i o n=X$ ', where $\mathrm{X}$ refers to the desired high-resolution cutoff in $\AA$.
In addition to QM-based X-ray refinement, conventional (i.e. non-QM) refinements using the same version of PHENIX were also performed for each case in order to explore the impact of the refinement method on the XModeScore results. The necessary CIF files for each protonation/tautomer state were generated using both the electronic Ligand Builder and Optimization Workbench (eLBOW) module (Moriarty et al., 2009), which generates restraints using quantum mechanics, and the Grade web server (http://grade.globalphasing.org), which produces Mogul CIFs based on coordinate data from the Cambridge Structural Database (CSD). The metal restraints around $\mathrm{Zn}$ were incorporated in the conventional refinements of the AZM structure as generated with the PHENIX program ReadySet!.

\subsection{XModeScore: scoring procedure}

The overall goal of XModeScore is to determine which protonation or tautomer form is found in the experimental structure. After refinement, each structure was scored based on a combination of metrics which take into account both the structural characteristics of the ligand and its fit within the active site, as well as quality indicators of its agreement with the crystallographic electron density. The local ligand strain energy (SE) serves as an important quality indicator of protein-ligand structures because it shows how much strain the ligand must accept to bind to the protein. The SE or $E_{\text {Strain }}$ is defined as the difference between the energy of the isolated ligand conformation and the protein-bound ligand conformation and is computed (Fu et al., 2012) according to

$$
E_{\text {Strain }}=E_{\text {SinglePoint }}-E_{\text {Optimized }}
$$

where $E_{\text {SinglePoint }}$ is the single-point energy computed for the ligand X-ray geometry and $E_{\text {Optimized }}$ is the energy of the optimized ligand that corresponds to the local minimum. The strain energies in this work have been calculated using the

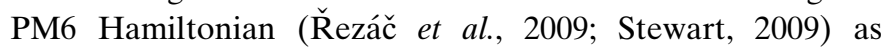
implemented in DivCon.

The experimental quality indicator component of XModeScore is a measure of the model accuracy or how well the model would have predicted the data. The generally accepted quality metric of the X-ray electron (or neutron) density is the real-space correlation coefficient (RSCC; Rupp, 2009). The RSCC reflects the degree of correspondence between the experimental (observed) and calculated electron densities. However, as argued previously (Tickle, 2012), the RSCC correlates both with the accuracy and with the precision of the structure model, and it is not possible to say to what extent the RSCC reflects the accuracy of a given model owing to the variable contribution from the precision component.

On the other hand, the real-space difference density $Z$ score for a point difference density value (Tickle, 2012) defined in (2) provides a more sophisticated quality indicator since it measures the accuracy of the model,

$$
Z[\Delta \rho(\mathbf{r})]=\frac{\Delta \rho(\mathbf{r})}{\sigma[\Delta \rho(\mathbf{r})]},
$$


where $\Delta \rho(\mathbf{r})$ is the difference density at the coordinate vector r expressed as the real Fourier transform (Read, 1986),

$$
\begin{aligned}
\Delta \rho(\mathbf{r})= & \left(2 / V_{\text {cell }}\right) \sum_{\mathbf{h}} c(\mathbf{h})\left[m(\mathbf{h}) F_{\text {obs }}(\mathbf{h})-D(\mathbf{h}) F_{\text {calc }}(\mathbf{h})\right] \\
& \times \cos [2 \pi \mathbf{r} \cdot \mathbf{s}(\mathbf{h})-\varphi(\mathbf{h})] .
\end{aligned}
$$

Here, the sum is over observed reflections with index vector $\mathbf{h}$ symmetry-expanded to a complete hemisphere in reciprocal space, $F_{\text {obs }}(\mathbf{h})$ and $F_{\text {calc }}(\mathbf{h})$ are the observed and calculated structure-factor amplitudes, respectively, $\varphi(\mathbf{h})$ is the phase calculated from the model, $c(\mathbf{h})$ is the centricity factor ( 1 for centric reflections or 2 for acentric reflections), $m(\mathbf{h})$ is the expected cosine of the phase error, $D(\mathbf{h})$ is a correction factor for errors in the model and $\mathbf{s}(\mathbf{h})$ is the scattering vector.

In (2), $\sigma[\Delta \rho(\mathbf{r})]$ is the standard deviation of the difference density, which is the standard measure of random error and is therefore a pure precision metric. The $Z$ score of the difference density is a measure of the residual nonrandom error and thus is a pure accuracy metric. However, a single minimum or maximum value of the difference density might not be statistically sound, as it is easy to overinterpret the significance of such a $Z$ score (Tickle, 2012). Difference density $Z$ values should approach a normal distribution of random errors with zero mean and unit standard deviation as the quality of the model improves, and the presence of significant positive or negative peak outliers that deviate from the expected distribution indicate problems with the model. Therefore, rather than using the point density at the atom center, or a single minimum or maximum value for each atom taken over all grid points covering the atom, it is more reliable to compute the standard chi-square $\left(\chi^{2}\right)$ statistic for a subset of the absolute negative values, and similarly for the positive values, of the density at the grid points covering an atom, assuming independent and identically distributed (iid) random variables. In each case the selected subset starts at the $k$ th value in increasing order of magnitude,

$$
\chi_{k}^{2}=\sum_{i=k}^{N} x_{(i)}^{2}
$$

where $x_{(i)}$ is the $i$ th normal order statistic (i.e. postulating the null hypothesis of a normal distribution) of the $|Z(\Delta \rho)|$ scores for the negative and positive values, respectively [i.e. in each case the $i$ th value after sorting each array of $|Z(\Delta \rho)|$ values in increasing order of magnitude].

Thus, all such grid-point density values become potentially relevant during the evaluation of the ZDD metric, which is a measure of the difference density of an atom that takes into account its variation over the entire atomic volume $(6 a$ and $6 b$ ). Clearly, we do not know a priori which of the density values are significant: if we choose too few we may lose information, but if we choose too many and add noise then $\chi^{2}$ will lose significance. Therefore, it is reasonable to sum only the subset of values of $x_{(i)}^{2}$ that maximizes the probability $p_{\max }$ over $k$,

$$
\begin{aligned}
p_{\max }= & \max _{k} P\left(\chi_{k}^{2} \leq \sum_{i=k}^{N} x_{(i)}^{2}\right) \\
\simeq & \max _{k} P\left[\frac{1}{2} \sum_{i=k}^{N} x_{(i)}^{2} ;(N+1-k) / 2\right] \\
& \times I\left\{2 \Phi\left[x_{(k)}\right]-1 ; k-1, N+1-k\right\},
\end{aligned}
$$

where the function $P$ is the lower normalized gamma function representing the cumulative distribution function (CDF) of $\chi_{k}^{2}$. In practice, the $\mathrm{CDF}$ is computed as the complement $(1-P)$ to avoid problems with numerical precision for values of the function $P$ near unity, i.e. the most relevant values for the present purposes. The second function, $I$, which is also computed as the complement in practice, is the normalized incomplete beta function (CDF of a normal order statistic; Gibbons \& Chakraborti, 2010) which accounts for the 'multiple comparisons' correction (Yuriev \& Ramsland, 2013). It is worth remarking that in the special case of $k=N$, where the maximal probability $p_{\max }$ occurs when only the single maximum absolute density value is used, the function $I$ becomes the Dunn-Šidák correction (Šidák, 1967). Another special case occurs for $k=1$, where the maximal probability occurs when all density values are used. In this case there is no 'multiple comparisons' correction, so the function $I$ is then exactly 1 and the combined function reduces to the CDF of $\chi^{2}$ for $N$ degrees of freedom, as expected. In this way, the probability $p_{\max }$ makes no assumptions about the spatial distribution of significant grid-point values in the vicinity of an atom (e.g. whether there is a single sharp maximum, a broad maximum or multiple maxima). Rather, the value of $p_{\max }$ adapts to the actual distribution and attempts to quantify the probability that the distribution of grid-point values could not have arisen purely from random variations.

ZDD is evaluated as the two-tailed normal $Z$ score corresponding to the maximal value $p_{\max }$ over $k$ of the cumulative probability of $\chi_{k}^{2}$ derived from (4) and (5),

$$
\mathrm{ZDD}=\Phi^{-1}\left[\left(1+p_{\max }\right) / 2\right]
$$

or

$$
\mathrm{ZDD}=-\Phi^{-1}\left[\left(1-p_{\max }\right) / 2\right] .
$$

Here, the function $\Phi$ is the CDF of the normal distribution [so $2 \Phi(|Z|)-1$ is the CDF of the half-normal distribution of the absolute value of a normal variate $Z]$ and $\Phi^{-1}$ is the inverse function (i.e. the value of $Z$ corresponding to a given probability). The form ( $6 b)$ is preferred because the complement (1 $-p_{\max }$ ) of the probability was calculated in the previous step.

ZDD also depends on the radius $r_{\max }$ enclosing the atomic density grid points; this is determined from the radius integral

$$
R_{\text {atom }}=\int_{0}^{r_{\max }} \rho(r) \mathrm{d} r .
$$

The radius $r_{\max }$ corresponds to the value of the radius integral $R_{\text {atom }}$ that is $95 \%$ of the theoretical value at infinite radius (Tickle, 2012). For this purpose, the calculated atomic density function $\rho(r)$ is determined from the spherically averaged real Fourier transform according to 
Table 1

Scoring results for possible tautomeric structures of the ligand AZM in PDB entry 3hs4.

The correct tautomeric state data are highlighted in bold.

\begin{tabular}{|c|c|c|c|c|}
\hline & SE & RSCC & ZDD & XModeScore \\
\hline \multicolumn{5}{|c|}{ Structure $3 \mathrm{hs} 4$} \\
\hline 3 & 5.55 & 0.989 & 12.8 & 2.72 \\
\hline 2 & 8.89 & 0.978 & 24.9 & -0.74 \\
\hline 1 & 10.8 & 0.975 & 27.2 & -1.98 \\
\hline \multicolumn{5}{|c|}{ Resolution $1.4 \AA$} \\
\hline 3 & 5.89 & 0.989 & 9.42 & 2.77 \\
\hline 2 & 9.31 & 0.981 & 18.1 & -0.88 \\
\hline 1 & 10.1 & 0.978 & 20.9 & -1.88 \\
\hline \multicolumn{5}{|c|}{ Resolution $1.6 \AA$} \\
\hline 3 & 6.01 & 0.987 & 7.87 & 2.72 \\
\hline 2 & 8.71 & 0.980 & 14.3 & -0.70 \\
\hline 1 & 9.75 & 0.978 & 16.8 & -2.02 \\
\hline \multicolumn{5}{|c|}{ Resolution $1.8 \AA$} \\
\hline 3 & 6.13 & 0.988 & 6.18 & 2.13 \\
\hline 2 & 6.46 & 0.982 & 11.4 & 0.40 \\
\hline 1 & 9.24 & 0.978 & 14.6 & -2.53 \\
\hline \multicolumn{5}{|c|}{ Resolution $2.0 \AA$} \\
\hline 3 & 5.58 & 0.989 & 6.56 & 2.68 \\
\hline 2 & 8.74 & 0.982 & 12.3 & -1.24 \\
\hline 1 & 7.86 & 0.975 & 15.6 & -1.45 \\
\hline \multicolumn{5}{|c|}{ Resolution $2.2 \AA$} \\
\hline 3 & 5.77 & 0.989 & 6.17 & 2.77 \\
\hline 2 & 7.73 & 0.981 & 10.8 & -1.31 \\
\hline 1 & 8.35 & 0.984 & 10.0 & -1.47 \\
\hline \multicolumn{5}{|c|}{ Resolution $2.5 \AA$} \\
\hline 3 & 5.40 & 0.989 & 7.65 & 2.47 \\
\hline 2 & 8.20 & 0.986 & 8.62 & -0.04 \\
\hline 1 & 11.1 & 0.984 & 9.48 & -2.43 \\
\hline \multicolumn{5}{|c|}{ Resolution $2.8 \AA$} \\
\hline 3 & 5.45 & 0.984 & 9.67 & 2.80 \\
\hline 2 & 8.25 & 0.984 & 10.2 & -1.39 \\
\hline 1 & 8.74 & 0.982 & 10.1 & -1.41 \\
\hline \multicolumn{5}{|c|}{ Resolution $3.0 \AA$} \\
\hline 2 & 8.02 & 0.983 & 11.3 & 0.49 \\
\hline 3 & 5.67 & 0.981 & 11.9 & 0.01 \\
\hline 1 & 8.61 & 0.983 & 11.5 & -0.50 \\
\hline
\end{tabular}

$$
\rho(r)=\left(\frac{8 n}{r}\right) \int_{s_{\min }}^{s_{\max }} f(s) \exp \left(-B s^{2}\right) \sin (4 \pi r s) s \mathrm{~d} s
$$

where $n$ is the fractional occupancy of the atom, $s_{\min }=0.5 / d_{\max }$ depends on the maximum $d$-spacing (or low-resolution limit) $d_{\max }$ and $s_{\min }=0.5 / d_{\max }$ similarly on the minimum $d$-spacing (or high-resolution limit) $d_{\min }, f(s)$ is the atomic scattering factor for X-rays as a function of $s$ and $B$ is the isotropic displacement parameter ( $B$ factor). Thus, the width of the atomic density function (and hence $r_{\max }$ ) will be greater at lower resolution and for larger values of the $B$ factor, in line with what one expects to see in an electron (or neutron) density map. Where the densities of adjacent (i.e. bonded) atoms overlap, the densities at the grid points in the electrondensity map are partitioned in proportion to the atomic densities calculated from (8).

To avoid oversampling the density values at the grid points, which would invalidate the iid assumption made above, the set of density values are resampled according to the ShannonNyquist theorem (Shannon, 1949). This theorem states that the density values at the grid points are statistically independent when the sampling interval is at least $d_{\min } / 2$, where $d_{\min }$ is the minimum $d$-spacing of the data used in the computation of the map. Typically, maps are sampled at an interval of not more than $d_{\min } / 4$ for accurate interpolation and to avoid missing important features, so the map would need to be resampled for the statistical calculations at about every second grid point in each direction. However, since resampling the map in three dimensions might lose information such as significant outliers, the density values are sorted by increasing value as a one-dimensional array and then resampled, keeping only a fraction (e.g. 1/8).

The set of negative density values then yields a metric that we call ' $Z D D-$ ' and the set of the positive densities yields the metric 'ZDD+'. Therefore, the effects of negative difference density, owing to incorrectly positioned atoms, and positive difference density (perhaps owing to an incorrectly typed atom) can be separately identified. The ZDD- and ZDD+ metrics are also taken together to give a final combined ZDD metric defined as

$$
\mathrm{ZDD}=\max (\mathrm{ZDD}-, \mathrm{ZDD}+)
$$

The lowest ZDD in the series of ligand tautomeric forms allows us to choose the best form or protonation state that demonstrates the closest match with experimental density. Then, with both QM-SE and ZDD in hand, the overall score of the tautomer form $i$ can be calculated according to

$$
\text { Score }_{i}=-\left(\frac{\mathrm{ZDD}_{i}-\mu_{\mathrm{ZDD}}}{\sigma_{\mathrm{ZDD}}}+\frac{\mathrm{SE}_{i}-\mu_{\mathrm{SE}}}{\sigma_{\mathrm{SE}}}\right),
$$

where $\mu$ is the mean value and $\sigma$ is the standard deviation of the corresponding array of data (ZDD or SE). For example, the SE array contains SE values for all tautomers included in the calculations. The highest $\mathrm{Score}_{i}$ corresponds to the best tautomeric form $i$ that fits both the SE and the ZDD criteria.

\section{Results}

3.1. The protonation state of AZM bound to human carbonic anhydrase II: PDB entry 3 hs 4 at $1.1 \AA$ resolution

Human carbonic anhydrase II (HCA II), which catalyzes the hydration/dehydration of carbonates, is involved in<smiles>CC(=O)Nc1nnc(S(N)(=O)=O)s1</smiles>
1<smiles>CC(=O)Nc1nnc(S(N)(=O)=O)s1</smiles>

2<smiles>CC(=O)Nc1nnc(S(N)(=O)=O)s1</smiles>

Figure 1 3

Possible binding modes of the drug AZM. 
numerous metabolic processes including $\mathrm{CO}_{2}$ transport and $\mathrm{pH}$ regulation and is therefore considered to be an important target for drug design (Krishnamurthy et al., 2008; Merz \& Banci, 1997). The drug acetazolamide (AZM), sold under the name Diamox, is a high-affinity inhibitor of HCA II that is used to efficiently treat a number of medical conditions (United States Pharmacopeia, 1995; Moldow et al., 1999) such as altitude sickness, hypertension and glaucoma. It binds to the $\mathrm{Zn}$ atom of the enzyme via the $\mathrm{N}$ atom of the sulfonamido

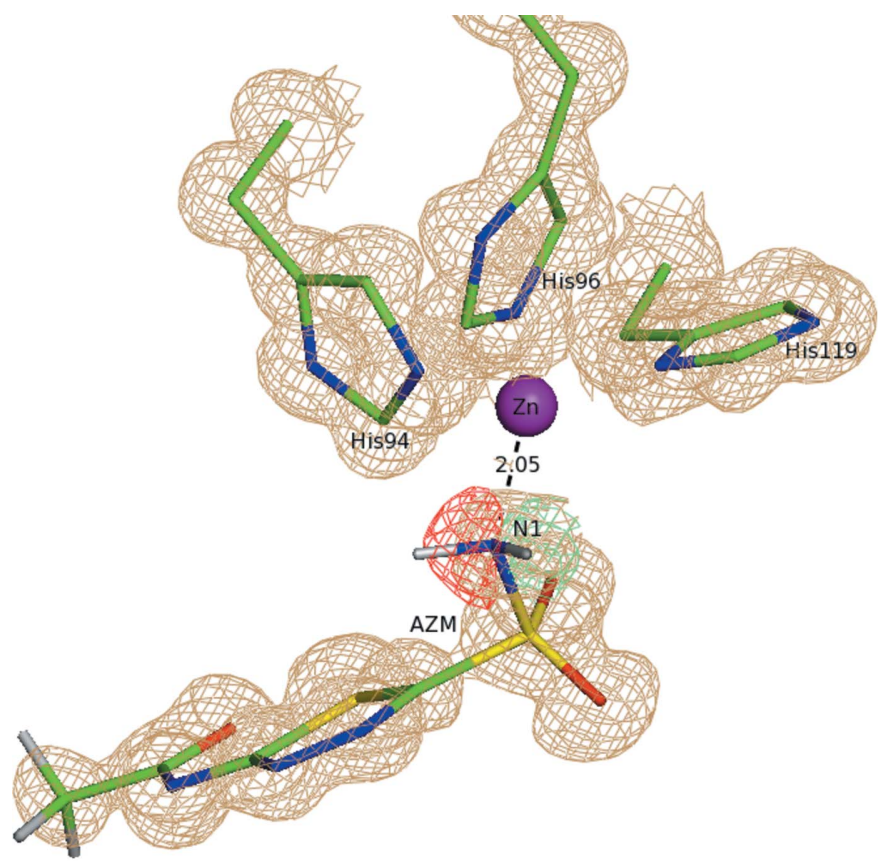

(a)

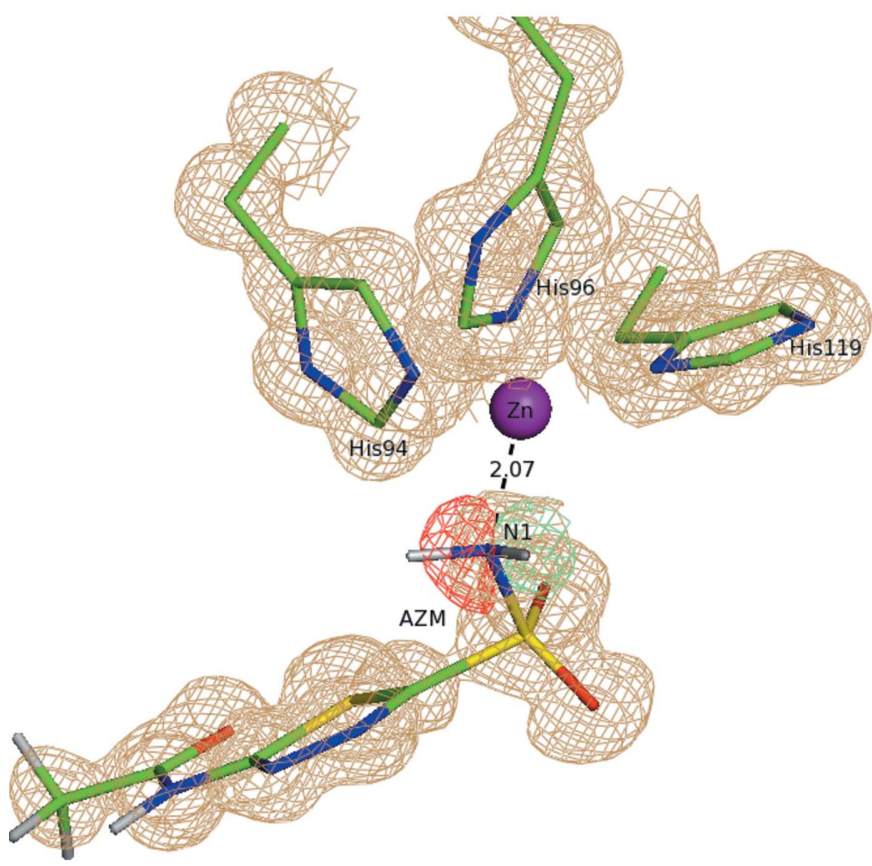

(b) group. $\mathrm{Zn}$ is located in the catalytic center of HCA II and adopts a tetrahedral coordination, making coordination bonds to $\mathrm{N}$ atoms of His94, His96 and His119. AZM can exist in two protonation states and several tautomeric forms, which are depicted in Fig. 1. However, even high-resolution X-ray diffraction studies have failed to determine which form of AZM is actually involved in the enzyme interaction (Sippel et al., 2009; Fisher et al., 2012). Conventional protonationdetermination methods, such as analysis of the bond-length distribution (Ahmed et al., 2007), also failed in the case of AZM (Fisher et al., 2012). It was only with a recent neutron diffraction study (Fisher et al., 2012) that it was established that AZM exists in form $\mathbf{3}$, which includes the negatively charged sulfonamido $\left(\mathrm{SO}_{2} \mathrm{NH}\right)$ group bound to zinc.

We challenged XModeScore with three structures which include three possible forms of AZM beginning with PDB entry 3hs4 (Sippel et al., 2009; Table 1 and Fig. 2). The results indicate that form $\mathbf{3}$, the correct protonation state according to the neutron diffraction experiment, is indeed the superior form, dominating in both components of scoring. There is a significant difference in the score for form 3 (2.72) and the score for the second-best option $(-0.74)$, which corresponds to form 2. It is important to note that the ZDD of form $\mathbf{3}$ is almost half that of the other two forms, which suggests that structure of tautomer $\mathbf{3}$, with the negative charge on the N1 atom bound to zinc, is more consistent with the experimental structure amplitudes than are the forms with an amino group at this position. The difference density maps obtained after QM refinement show that the negatively charged N1 plays a crucial role in binding to HCA II. In particular, large negative/ positive peaks of the difference density are seen around the

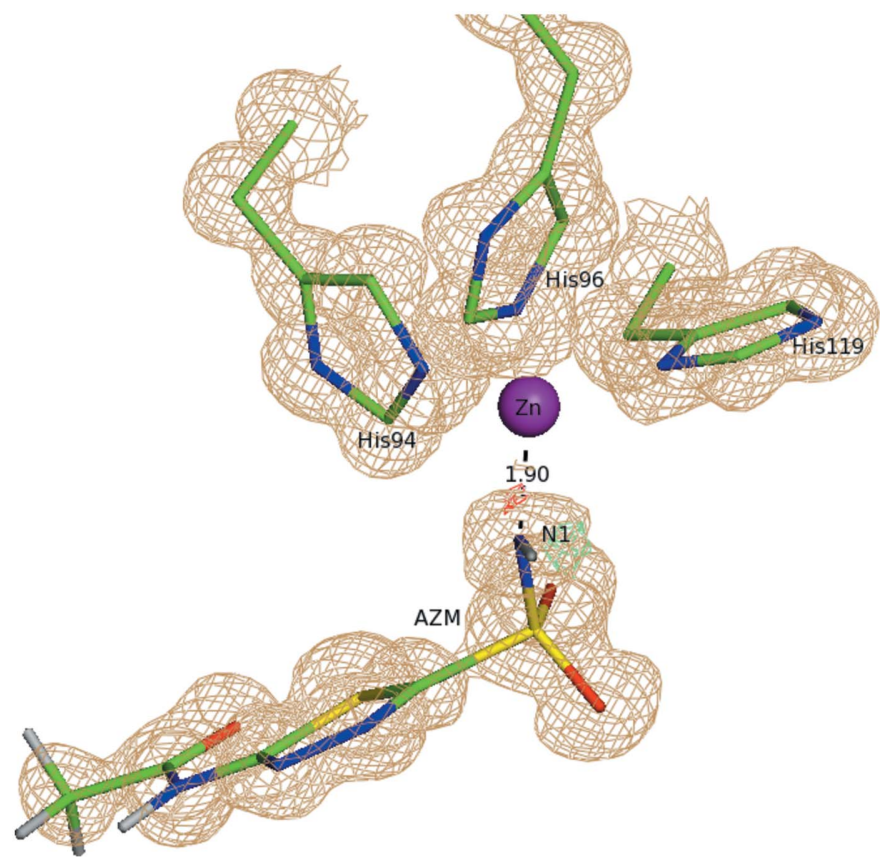

(c)

Figure 2

The coordination sphere of $\mathrm{Zn}$ in the catalytic center of HCA II with a bound AZM molecule in three alternative binding modes $\mathbf{1}(a), \mathbf{2}(b)$ and $\mathbf{3}(c)$ after QM refinement of the PDB structure 3hs4. The difference density around the key N1 atom of the sulfonamido group of AZM is contoured at the $3.5 \sigma$ level. 
Table 2

Geometry and electron-density characteristics (ZDD-/ZDD+) of the N1 atom of AZM directly bound to the $\mathrm{Zn}$ atom in the structure with PDB code 3 hs 4 .

The correct tautomeric state data are highlighted in bold.

\begin{tabular}{llll}
\hline 3hs4 AZM tautomers & 1 & 2 & $\mathbf{3}$ \\
\hline Zn-AZM N1 (̊) & 2.05 & 2.07 & $\mathbf{1 . 9 0}$ \\
AZM N1 ZDD-/ZDD+ & $14.27 / 20.45$ & $14.58 / 21.64$ & $\mathbf{4 . 3 5 / 4 . 6 6}$
\end{tabular}

$\mathrm{N} 1$ atom for the tautomers $\mathbf{1}$ and $\mathbf{2}$ and effectively explain the larger magnitude of ZDD observed for the former tautomer states compared with form 3. Furthermore, analysis of the bond-length distribution around zinc after $\mathrm{QM}$ refinement (Table 2) shows that the $\mathrm{Zn}-\mathrm{AZMN} 1$ bond in tautomer $\mathbf{3}$ $(1.90 \AA)$ is much shorter than the length of this coordination bond in the other two tautomers ( 2.05 and $2.07 \AA$ ) and is also shorter than the average length of 2.00 (2) $\AA$ for the $\mathrm{Zn}-\mathrm{N}$ bond type (Harding, 1999). Nevertheless, such a binding geometry of the ligand AZM with a shortened $\mathrm{Zn}-\mathrm{N} 1$ bond seen in form 3 agrees much better with the experimental data. Specifically, the atomic ZDD for the N1 atom in form $\mathbf{3}$ is fourfold lower than the corresponding values observed for the tautomers with a $\mathrm{Zn}-\mathrm{NH}_{2}$ bond.

\subsection{Effect of resolution truncation on the predictability of the tautomer by XModeScore}

Generally, at lower resolution less detail about the crystal model is revealed from experimental data and these experimental data are less sensitive to the model nuances. Hence, the resolution of data sets may affect not only the absolute values of ZDD but also the difference in tautomer scores. The latter is crucial as it determines the ability to distinguish the top tautomeric form from the rest of the candidates in XModeScore. To determine how the resolution affects the predictability of XModeScore, we carried out stepwise truncation of the original data set $3 \mathrm{hs} 4$ followed by repetition of the scoring protocol at each resolution level.

Through the truncation of the original high-resolution data set 3 hs 4 , one can explore how well the method maintains its predictive power over decreasing resolution (Table 1) while controlling for inconsistencies in experimental conditions $(\mathrm{pH}$, temperature, solvent and so on) between native high- and lowresolution structures. For the 3 hs 4 refinement, XModeScore is able to remain predictive until a low resolution of $3.0 \AA$ is achieved. $\triangle \mathrm{ZDD}$ is the change in ZDD between one tautomer and another tautomer and is an indication of how well the ZDD will differentiate between the tautomers. At resolutions higher than $1.8 \AA$ tautomer 2 exhibits a high value of $\triangle \mathrm{ZDD}$. However, $\triangle \mathrm{ZDD}$ decreases towards zero when the resolution decreases to $3.0 \AA$ as molecular details of the structure are becoming smeared, as discussed above. At a resolution of $2.8 \AA \Delta$ ZDD is close to zero, which prevents a reliable distinction between forms $\mathbf{2}$ and $\mathbf{3}$ based on the density score alone. Generally speaking, ZDD tends to diminish in magnitude and equalize between tautomers at lower resolutions. On the other hand, when considering the overall $\Delta \mathrm{XModeScore}$, the value changes far less and is fairly flat, suggesting that even if the experimental density deteriorates with the resolution, the second component (e.g. ligand strain) significantly augments the deteriorating $\triangle \mathrm{ZDD}$ value and leads to the selection of the correct tautomer form at lower resolutions. It is notable that the $\triangle \mathrm{RSCC}$ function is the flat, virtually zero line. This relationship underscores the fact that the RSCC undergoes very little change between modes. This observation is consistent with the conclusion above that RSCC is not likely to be an appropriate metric for scoring. For clarity, these relationships are plotted in Fig. 3.

\subsection{AZM in PDB entry $4 \mathrm{k} 0$ s at $1.8 \AA$ resolution}

While using truncated data is an expedient and straightforward method of exploring predictability, a truncated highresolution data set still has much better quality in terms of the merging $R_{\text {merge }}$ factor of diffraction data, their completeness and redundancy, and of the mean signal-to-noise $(I / \sigma)$ ratio when compared with those of the native low-resolution data (Wlodawer et al., 2008). Therefore, we repeated our study on another structure of HCA II complexed with AZM (PDB entry $4 \mathrm{k} 0 \mathrm{~s})$ determined at the more modest resolution of $1.8 \AA$ A. Again, just as in the $3 \mathrm{hs} 4$ case, XModeScore found that tautomer $\mathbf{3}$ is the preferable tautomer according to both ZDD and SE components (Supplementary Table S1).

\subsection{The $8 \mathrm{HX}$ inhibitor in PDB entry 4n9s}

The enzyme urate oxidase is involved in the metabolism of purines, and to investigate the mechanism of action of urate oxidase the neutron diffraction structure of the enzyme in complex with uric acid monoanion (the inhibitor $8 \mathrm{HX}$ ) was determined (Oksanen et al., 2014; PDB entry 4n9m). In particular, the authors showed that the inhibitor is present in

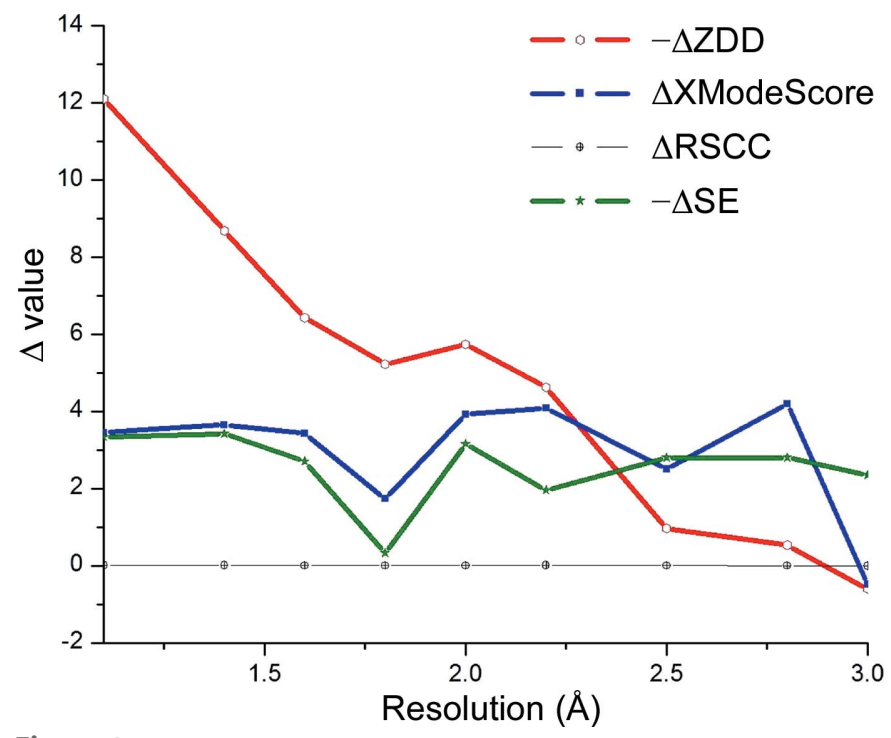

Figure 3

The differences in the raw values of ZDD, RSCC, strain energy (SE) and XModeScore for the AZM binding modes 3 and $\mathbf{2}$ plotted versus the resolution. 
Table 3

Scoring results for possible protonated states of Asp215 in PDB entry 2jjj.

The correct protonated state data are highlighted in bold.

\begin{tabular}{|c|c|c|c|c|}
\hline & SE & RSCC & ZDD & XModeScore \\
\hline \multicolumn{5}{|c|}{ Structure $2 \mathrm{jjj}$} \\
\hline 1 & 12.2 & 0.987 & 6.05 & 1.59 \\
\hline 2 & 9.08 & 0.977 & 12.3 & 0.24 \\
\hline 3 & 17.3 & 0.979 & 11.3 & -1.83 \\
\hline \multicolumn{5}{|c|}{ Resolution $1.4 \AA$} \\
\hline 1 & 12.7 & 0.990 & 3.66 & 1.33 \\
\hline 2 & 9.26 & 0.983 & 7.19 & -0.08 \\
\hline 3 & 17.3 & 0.987 & 5.36 & -1.25 \\
\hline \multicolumn{5}{|c|}{ Resolution $1.6 \AA$} \\
\hline 1 & 12.7 & 0.988 & 3.26 & 1.43 \\
\hline 2 & 9.29 & 0.980 & 6.15 & 0.01 \\
\hline 3 & 17.5 & 0.983 & 4.98 & -1.44 \\
\hline \multicolumn{5}{|c|}{ Resolution $1.8 \AA$} \\
\hline 1 & 12.9 & 0.989 & 2.66 & 1.09 \\
\hline 2 & 9.29 & 0.981 & 6.52 & -0.19 \\
\hline 3 & 17.5 & 0.987 & 3.68 & -0.90 \\
\hline \multicolumn{5}{|c|}{ Resolution $2.0 \AA$} \\
\hline 1 & 12.6 & 0.989 & 1.87 & 1.13 \\
\hline 2 & 9.24 & 0.982 & 4.94 & -0.23 \\
\hline 3 & 17.5 & 0.987 & 2.64 & -0.90 \\
\hline \multicolumn{5}{|c|}{ Resolution $2.2 \AA$} \\
\hline 1 & 12.4 & 0.986 & 2.92 & 0.97 \\
\hline 2 & 9.24 & 0.981 & 5.36 & -0.28 \\
\hline 3 & 17.4 & 0.987 & 3.10 & -0.68 \\
\hline \multicolumn{5}{|c|}{ Resolution $2.5 \AA$} \\
\hline 1 & 12.8 & 0.988 & 1.35 & 0.98 \\
\hline 2 & 9.48 & 0.986 & 2.38 & -0.28 \\
\hline 3 & 17.9 & 0.989 & 1.46 & -0.71 \\
\hline \multicolumn{5}{|c|}{ Resolution $2.8 \AA$} \\
\hline 2 & 9.52 & 0.987 & 1.90 & 0.03 \\
\hline 3 & 17.9 & 0.991 & 0.186 & 0.02 \\
\hline 1 & 13.1 & 0.99 & 1.23 & -0.06 \\
\hline
\end{tabular}<smiles></smiles>

Figure 4

The key lactam-lactim equilibrium for the ligand $8 \mathrm{HX}$ (Oksanen et al., 2014). the form of the 8-hydroxyxanthine monoanion $\mathbf{2 4}$ that exists in equilibrium with form 21 in solution (Fig. 4).

The neutron diffraction structure $4 \mathrm{n} 9 \mathrm{~m}$ revealed that the form $\mathbf{2 4}$ occurs in the crystal. Such a conclusion is supported by an unambiguous deuterium density peak near the $\mathrm{O}$ atom at position 8 , reflecting the hydroxyl group. The authors postulate that there is a water molecule near the hydroxyl $\mathrm{O}(8) \mathrm{H}$ observed in the neutron diffraction experiment that might stabilize form 24. However, a water molecule is capable of being both a donor and an acceptor of hydrogen bonds, and it is more likely to adapt to the solute (protein-ligand complex) rather than decisively determine its protonation state (Krieger et al., 2012). Since this water molecule is not seen in the relevant high-resolution X-ray structure $4 \mathrm{n} 9 \mathrm{~s}$, we believe that the hydrogen bond between N7 of the ligand and $\mathrm{N}-\mathrm{H}$ (backbone) of Thr57 observed for the symmetry-related protein molecule in the crystal favors an unprotonated $\mathrm{N} 7$ and hence the tautomer 24.

As many as 30 tautomer candidates of the two protonation states of $8 \mathrm{HX}$ were generated by WashMoleculeMOE (Supplementary Fig. S1), and XModeScore scores tautomer 24 at the top of the list based upon both scoring components (Supplementary Table S2). Comparing 24 (XModeScore 3.87) with its counterpart 21 (XModeScore 1.14) in the equilibrium shows the clear preference for the former. Additionally, the ZDD for $\mathbf{2 4}$ is lower (better) than the ZDD of $\mathbf{2 1}$ by 3.2 units. Truncating the resolution of the data, followed by QM refinement of the same set of tautomer structures, generally shows a similar trend until the low resolution of $3.0 \AA$ is reached: the tautomer $\mathbf{2 4}$ remains at the the top of the list, while form $\mathbf{1 7}$ is consistently at the bottom (Supplementary Table S2).

\subsection{The protonation state of the catalytic Asp215 in $2 \mathrm{jjj}$}

Aspartic proteinases are enzymes that are involved in many metabolic processes and are associated with the progression of a number of diseases, including AIDS (Cooper, 2002; Davies, $1990)$; in recent years, aspartic proteinases have received

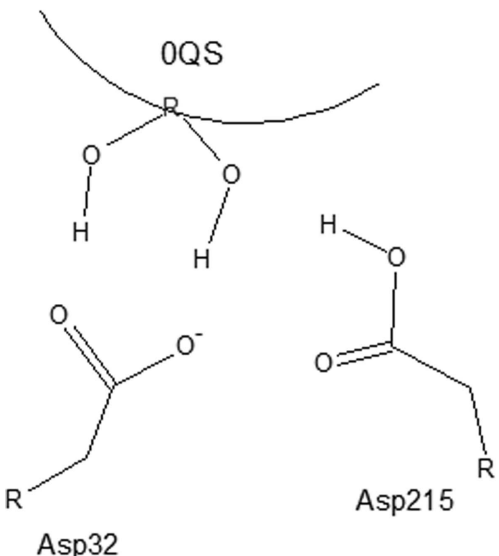

1
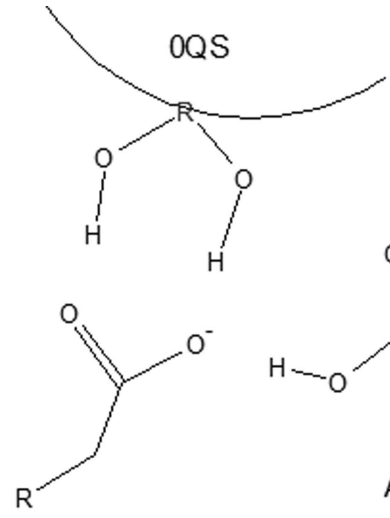

Asp32

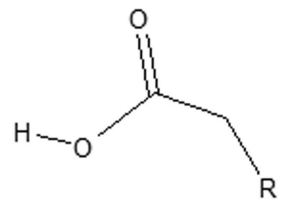

Asp215

2

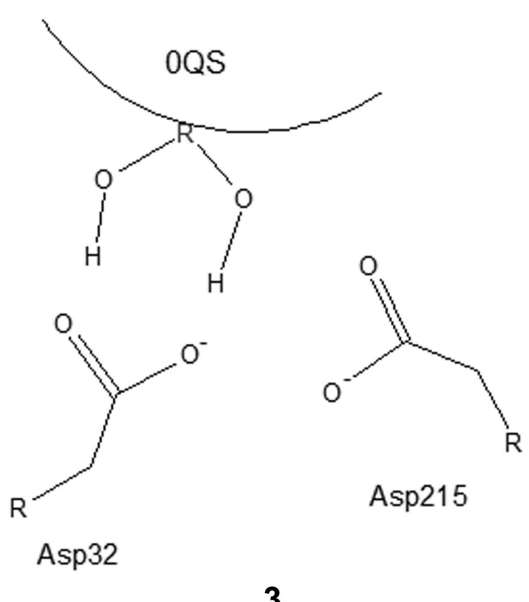

Figure 5

Three possible protonation states of Asp215 in the PD-135,040-aspartic proteinase structure. 
significant attention as promising drug-design targets (Eder et al., 2007). Several crystal structures of aspartic proteinases with a number of inhibitors are known, including the inhibitor PD-135,040 (PDB ligand 0QS; Supplementary Fig. S2), for which a neutron diffraction study has also been conducted (Coates et al., 2008). The preliminary X-ray study (Coates et al., 2003) demonstrated that the diol group of the ligand makes strong hydrogen bonds to two catalytic residues of the enzyme: Asp32 and Asp215. The neutron diffraction model (Coates et al., 2008) revealed that the outer O atom of Asp215 is protonated (structure $\mathbf{1}$ in Figs. 5 and 6).

For this case study, we generated the alternative structure 2 that has Asp215 protonated at the inner $\mathrm{O}$ atom, as well as structure 3 with a fully deprotonated Asp215 (Fig. 5). The XModeScore results for forms 1-3 after QM region refinement against the high-resolution X-ray structure 2jjj (Table 3) demonstrate an interesting interplay between the SE strain and ZDD components used within XModeScore. In this case, the SE strain of Asp215 rather than the ligand is considered as we vary the protonation states of the amino acid. In particular, protonation form $\mathbf{2}$ has the lowest strain energy, while the strain energy of the correct form $\mathbf{1}$ is about $3 \mathrm{kcal}$ higher.

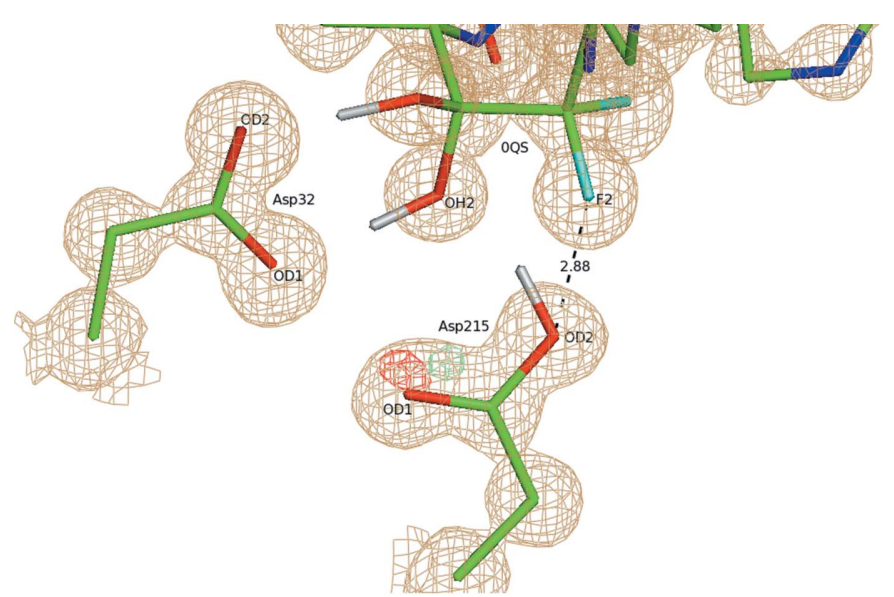

(a)

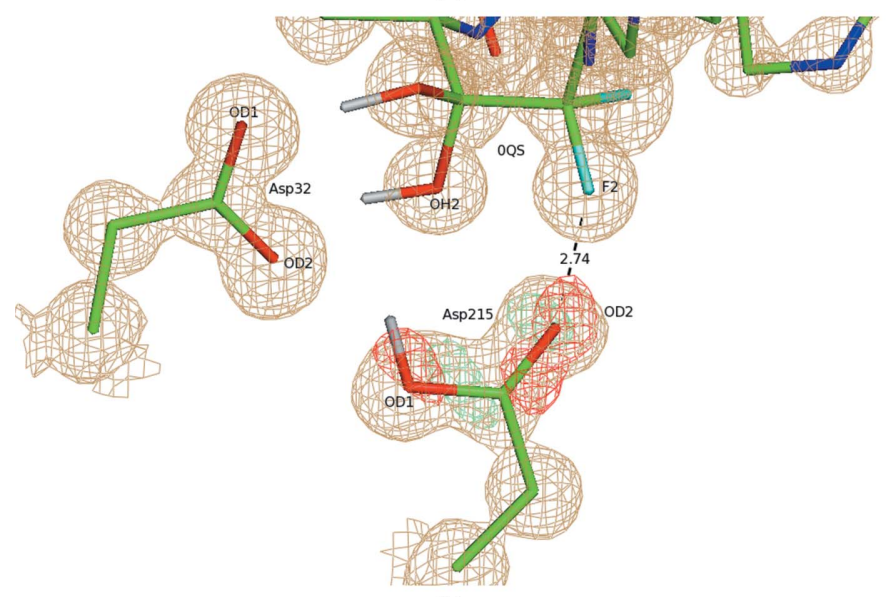

(b)
Table 4

Selected interatomic distances and electron-density characteristics (atomic ZDD-/ZDD+) for atoms of the catalytic residue Asp215 in PDB entry 2jjj.

The correct protonated state data are highlighted in bold.

\begin{tabular}{|c|c|c|c|}
\hline 2jjj Asp215 states & 1 & 2 & 3 \\
\hline Asp215 OD2-0QS F2 (̊̊)。 & 2.88 & 2.74 & 3.02 \\
\hline Asp215 OD2-0QS OH2 (Å)。 & 2.65 & 2.64 & 2.73 \\
\hline Asp215 OD1-Asp32 OH2 (̊) & 2.90 & 2.88 & 3.00 \\
\hline Asp215 OD1: ZDD-/ZDD+ & $5.98 / 3.76$ & $7.20 / 5.09$ & $7.78 / 5.35$ \\
\hline Asp215 OD2: ZDD-/ZDD+ & $3.66 / 1.23$ & $10.96 / 7.79$ & $12.89 / 8.10$ \\
\hline
\end{tabular}

Nevertheless, protonation form $\mathbf{1}$ of Asp215 is correctly scored as the best form owing to markedly better ZDD values. Such a low ZDD of form 1 can primarily be attributed to a positioning of the carboxyl group of Asp215 that is in much better agreement with experimental structure amplitudes compared with the other two protonation states. Indeed, difference density peaks around the carboxyl group are much lower for state 1 (Fig. 6). The location of the OD2 atom of Asp215 is particularly important. Its atomic ZDD in binding mode $\mathbf{1}$ is about fourfold better than that for states $\mathbf{2}$ and $\mathbf{3}$ (Table 4). The superimpositions of the atomic coordinates of Asp215 in all three forms after QM refinement (Fig. 7) revealed that OD2 in $\mathbf{1}$ is located in between the positions of this atom in the structures $\mathbf{2}$ and $\mathbf{3}$, which is also strongly correlated with the distance Asp215 OD2-0QS F2. Indeed, while the separation between the $\mathrm{F}$ atom of the inhibitor and Asp215 OD2 in $\mathbf{1}$ is $2.88 \AA$, the same distance is greater for form 3 by $0.14 \AA$ but is shorter for state 2 by $0.14 \AA$ (Table 4). Thus, the protonated atom OD2 in $\mathbf{1}$ apparently adopts an optimal location and even a relatively small shift such as $0.14 \AA$ in any direction, as seen in $\mathbf{2}$ and $\mathbf{3}$, leads to a dramatic increase of the atomic ZDD owing to an increase in the disagreement with the experimental density (Fig. 7 and Table 4).

At resolution truncations below $2.0 \AA$, the ZDD scores of forms $\mathbf{1}$ and $\mathbf{3}$ become similar. However, form $\mathbf{1}$ remains the

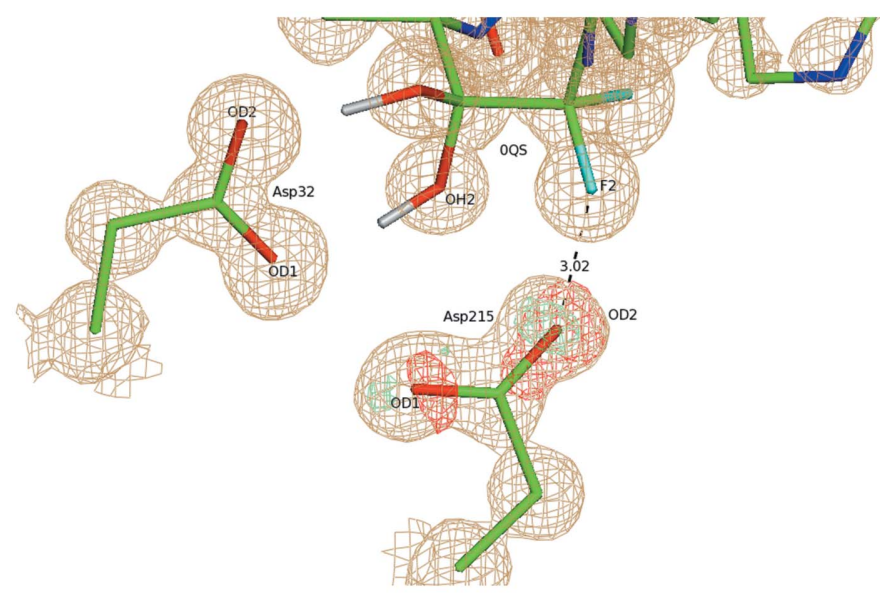

(c)

Figure 6

The catalytic center of aspartic proteinase with bound inhibitor $0 \mathrm{QS}$ in three alternative binding modes of Asp215, $1(a), 2(b)$ and $3(c)$, after QM refinement of PDB entry 2jjj. The difference density around the side chain of the key catalytic residue Asp215 is contoured at the $3.5 \sigma$ level. Only selected atoms of OQS are shown for clarity. 
top structure because its strain energy is lower than that of the unprotonated Asp215 form 3. When the data are truncated, this relationship is maintained until the resolution reaches $2.8 \AA$ and the scoring model no longer predicts the correct structure 1. Overall, the plots of $\Delta$ values in Fig. 8 for the structure 2jjj look similar to the same plot for the AZMbinding modes (Fig. 3). In particular, the $\triangle$ ZDD significantly decreases with resolution, while the $\Delta$ XModeScore function exhibits an essentially flat trend up to $2.8 \AA$ resolution. Generally, the plots in Figs. 3 and 8 confirm the universal nature of the XModeScore concept.

\section{Discussion}

In order to properly guide SBDD efforts, it is necessary to identify the correct tautomer/protomer state of the molecule in the bound state (Martin, 2009; Pospisil et al., 2003). The building blocks of common drug and drug-candidate small molecules include 5,6-membered heterocycles and various functional groups that make proton migration from one part of the molecule to another possible. Prototropy or proton-shift tautomerism represents the most common type of molecular rearrangement relevant to SBDD. Keto-enol, imine-enamine and other equilibrium types lead to hydrogen transfer between hydrogen-donor groups (e.g. $-\mathrm{OH},-\mathrm{NH} 2)$ and hydrogen-acceptor atoms (e.g. $=\mathrm{O},=\mathrm{N}-$ ) (Warr, 2010). While the tautomerism changes neither the molecular formula

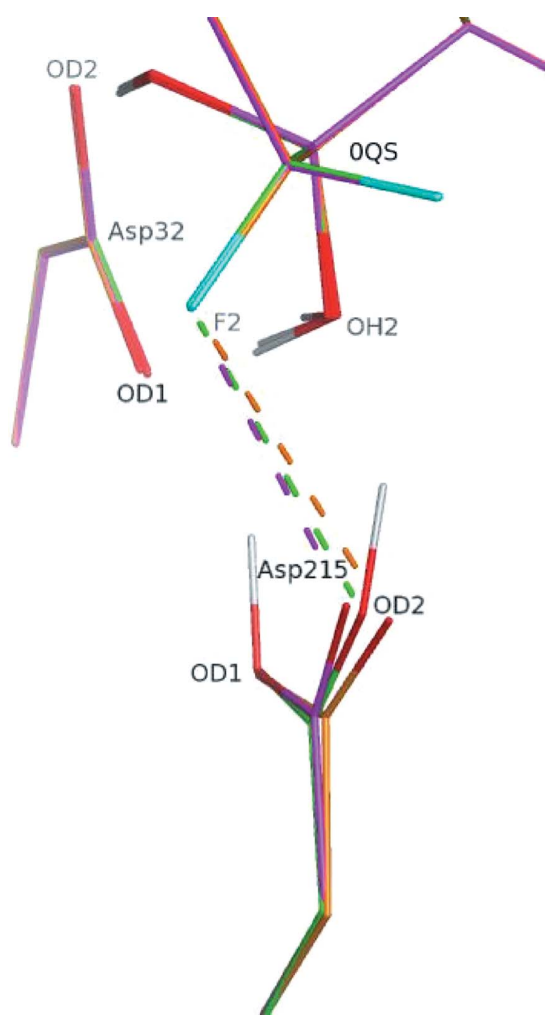

Figure 7

Superimposition of the key residues of the aspartic proteinase structure (PDB entry 2jjj) after QM refinements of three alternative binding modes of Asp215: 1 (green), 2 (magenta) and $\mathbf{3}$ (orange). Only selected atoms of OQS are shown for clarity. The key distances are listed in Table 4. nor the molecular charge, each tautomer is a distinct chemical structure with unique physico-chemical properties. The key point is that different tautomers exist in an equilibrium in solution where the ratio between possible states is affected by the $\mathrm{pH}$, temperature, concentration, ionic strength and other factors (Raczyńska et al., 2005). The general view is that protein receptors are capable of selectively binding a certain tautomeric form or forms from the mixture of several possible states (Pospisil et al., 2003). For example, the antibiotic tetracycline can exist and react in one of 64 possible tautomeric forms adapting to various chemical environments (Duarte et al., 1999). A growing body of evidence indicates that sometimes an unexpected tautomer form, or a form which does not correspond to the energy minimum of the tautomer set in vacuum, is found to react with the protein receptor (Martin, 2009).

The limitations of the current experimental techniques used for structure determination, where even at the extremely high resolution of $0.66 \AA$ only $54 \%$ of all $\mathrm{H}$ atoms are revealed (Howard et al., 2004), make it difficult to determine these states. As an alternative to X-ray crystallography, neutron diffraction is considered to be a unique technique that allows experimental determination of hydrogen positions in crystal structures at resolutions much lower than those used to reveal atomic details (Blum et al., 2009; Katz et al., 2006). However, owing to the limitations of neutron diffraction such as a reliance on large crystals, the necessity of deuterium exchange, the limited availability of sources of neutron radiation and difficulties in the refinement of $\mathrm{H}$ atoms with negative scattering length, neutron diffraction is of only limited utility in SBDD. In fact, it is notable that, as of June 2015, the overall number of structures determined using neutron diffraction available in the PDB remains at 88 versus the total of 97297 $\mathrm{X}$-ray structures.

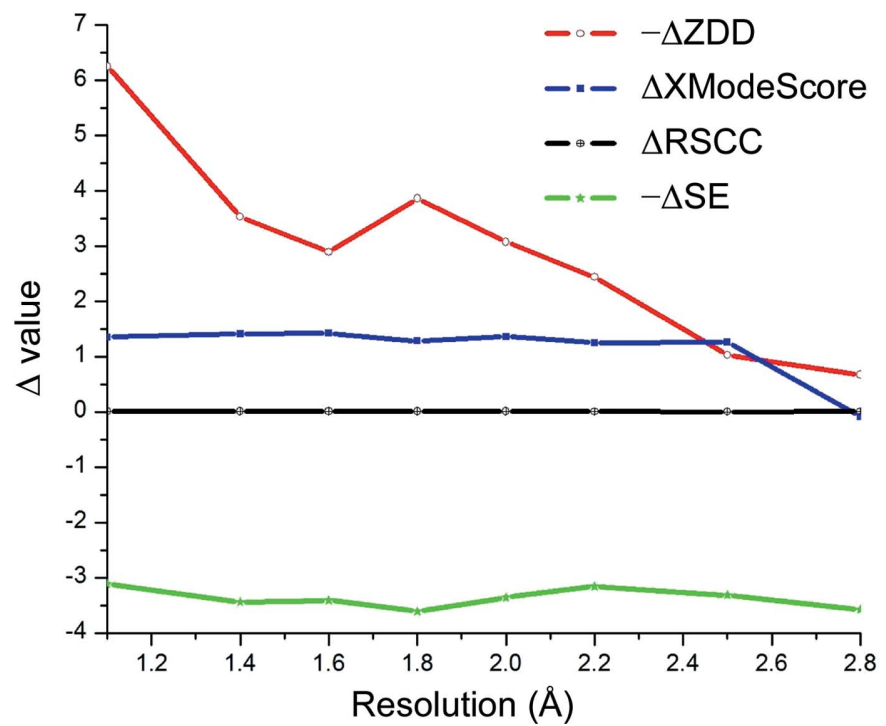

Figure 8

The differences in the raw values of ZDD, RSCC, strain energy (SE) and XModeScore for Asp215 binding modes $\mathbf{1}$ and $\mathbf{2}$ plotted versus resolution for PDB entry 2jjj. 
We have found that XModeScore is able to determine the protonation state of ligands and catalytic residues using routine X-ray crystallographic data with a level of accuracy that is only achieved in neutron diffraction studies coupled with high-resolution X-ray structures. Even when XModeScore is challenged with truncated or low-resolution (e.g. 2.5$3.0 \AA$ ) X-ray data, it is still observed to be predictive. The XModeScore method involves the QM X-ray refinement of a set of macromolecular structures containing all likely tautomer/protomer forms or binding modes, followed by a rigorous statistical analysis of difference electron-density maps around each candidate form coupled with computation of its QM strain. This approach allows us to choose the best tautomer based on a combination of energetics and of agreement between model and experimental density. Yu et al. (2006) have proposed a similar setup when a set of several protein structures with different protonation states of three key residues is refined with the CNS package using the QM/ MM protocol as described elsewhere (Yu et al., 2005). After each refinement, the relative stabilities of these protonation states were evaluated from thermodynamic cycles using energies from additional single-point semiempirical DivCon calculations. The key advantage of XModeScore over the above procedure (Yu et al., 2006) is that it directly employs the experimental electron density to judge the bound protomer.

In order to validate the applicability of XModeScore in the present work, we considered several key case studies. As the first example, for many years the correct binding form of the drug acetazolamide (AZM) in human carbonic anhydrase II was uncertain (Lesburg et al., 1997; Sippel et al., 2009) and the correct form was only unambiguously established by a rigorous joint neutron diffraction/X-ray study in 2012 (Fisher et al., 2012). With XModeScore, the same conclusion was reached utilizing the $\mathrm{X}$-ray data alone and it chose the correct tautomeric form over two other possible states of AZM by a wide margin (Table 1). At the structural level, the difference between the correct form $\mathbf{3}$ and the incorrect binding modes is primarily attributed to shortening of the $\mathrm{Zn}-\mathrm{N}$ coordination bond between the $\mathrm{N}$ atom of the AZM sulfonamido group and the cofactor of the enzyme seen in structure 3 (Table 2) after $\mathrm{QM}$ refinement. Notably, the $\mathrm{Zn}-\mathrm{N}$ distance of $1.9 \AA$ in $\mathbf{3}$ is shorter than the average distance of $2.00 \AA$ for the $\mathrm{Zn}-\mathrm{N}$ bond type (Harding, 1999) that is typically used for linkrestraint parameters in conventional refinement, suggesting that without a priori knowledge of the correct outcome it would be difficult for conventional, restraint-based refinement to come to the same conclusion. Nevertheless, such a short $\mathrm{Zn}-\mathrm{N}$ distance gives rise to the best agreement with the experimental data observed for binding mode 3 (Fig. 2 and Tables 1 and 2). This example underscores the importance of QM refinement as the indispensable step in successful scoring of the tautomer/protomer set. The key and unique advantage of QM refinement is to derive the geometry of protein-ligand systems objectively without making any a priori assumptions in the form of CIF dictionaries, fixed atom types, link restraints, coordination-sphere parameters or other 'usersupplied' characteristics (Borbulevych et al., 2014). When considering conventional refinement (Supplementary Tables S7 and S8) for 3hs4 mode $\mathbf{3}$ is still shown to be the best structure; however, the greater sensitivity of the QM-based refinement is apparent when one considers the spread of the ZDD score and XModeScore values across the three modes. In each case the 'spread' or discriminatory power of these indicators is much higher for the QM-based refinement. This is a crucial difference from scoring based on QM refinement, which demonstrates that QM-based refinement is better able to discriminate the correct mode $\mathbf{3}$ by a wide margin in both ZDD score and XModeScore, as was discussed above. One could speculate that this result could be owing to the fact that while the $e L B O W$-generated CIF for each tautomer captures the intramolecular conformational changes associated with protonation-state changes, the intermolecular interactions (e.g. electrostatics, polarization, charge transfer and so on) are not captured in the conventional refinement, and hence any impact of the active site on the different ligand protonation states are likewise missing. To further reinforce this point, we conducted another round of conventional refinement with AZM restraint CIFs provided by $M o g u l$, which are based on small-molecule crystallographic data from the Cambridge Structural Database (CSD; Supplementary Table S8). Clearly, Mogul restraints lead to lower strain energies of AZM, but the scoring results are essentially similar to those obtained with eLBOW restraints. Notably, the conventional refinements resulting from either type of CIF ceased to predict the correct tautomer after even a modest truncation of the resolution to $1.6 \AA$ (Supplementary Tables S7 and S8). This result shows that even high-quality stereochemical restraints cannot overcome the deficiency of the energy function used in conventional refinement, underscoring the superiority of $\mathrm{QM}$ refinement for XModeScore. Finally, for the sake of completeness, we also validated XModeScore with the AM1 Hamiltonian (Dewar et al., 1985; Supplementary Tables S3 and S6) and found that the scoring results are essentially similar to those with the PM6 Hamiltonian.

A large proportion of the available neutron diffraction experiments are focused on studies of enzymatic mechanisms in order to establish protonation states and the hydrogenbond network within the enzyme catalytic center (Blum et al., 2009; Tomanicek et al., 2013). The aspartic proteinase case study (Tables 3 and 4 and Figs. 5 and 6) clearly demonstrates that XModeScore is able to efficiently investigate the protonation state of the key catalytic residue Asp215 using X-ray data alone and to ultimately select the state which corresponds to that found in the neutron diffraction study reported by Coates et al. (2008). In this case, we discovered that there is a strong correlation between the distance Asp215 OD2-0QS F2 and the size of the difference density peaks or the magnitude of ZDD around the Asp215 OD2 atom (Table 4 and Fig. 7). A review (Müller et al., 2007) underscores the importance of fluorine substituents in SBDD since fluorine has unique properties which impact ligand affinity owing to polar hydrophobicity (Biffinger et al., 2004). The ability of fluorine to modulate ligand binding and even the immune response in peptide-based immunotherapy has been well documented 
(Gómez-Nuñez et al., 2008; Müller et al., 2007; Piepenbrink et al., 2009). Coates et al. (2008) do not elaborate on the possible fluorine effect in their manuscript; however, our results suggest that the protonation of Asp215 in the structure with the inhibitor 0QS (PD-135,040) might be modulated by the neighboring $\mathrm{F}$ atom rather than generally represent the mechanism of action of aspartic proteinase. Again, as in the AZM case, when considering the conventional refinement results provided in Supplementary Tables S9 and S10, the 'spread' of the XModeScore and ZDD score of the three Asp215 tautomers is an order of magnitude greater and the residue structural strain values are an order of magnitude lower for the QM-based refined models, suggesting that the QM values are likely to be more robust. This said, it is interesting to note that the ZDD of mode $\mathbf{1}$ is lower (better) than the ZDD of mode $\mathbf{1}$ observed in the QM-based refinement. Upon further exploration, the elevated ZDD score is associated with the backbone $\mathrm{O}$ and $\mathrm{C}$ atoms of Asp215, which can be attributed to the fact that SE methods such as PM6 overestimate the lengths of some protein backbone bonds (Borbulevych et al., 2014; Yu et al., 2005)

In the case study of the urate anion (ligand 8HX; Supplementary Table S2), XModeScore, using QM-based refinement, is able to select the correct tautomer from a large number of possible states using the very wide range of data resolution between 1.0 and $3.0 \AA$. Given the large number of possible tautomeric forms, it is interesting to consider the $\mathrm{p} K_{\mathrm{a}}$ of the structure. Uric acid has two $\mathrm{p} K_{\mathrm{a}}$ values (5.4 and 9.8) that are relevant in the physiological $\mathrm{pH}$ range considered (Simic \& Jovanovic, 1989). As a result, this compound exists predominantly as a monoanionic form. However, this monoanion can undergo lactam-lactim tautomerism as shown in Fig. 4 and it can exist in a number of other anionic tautomeric forms (Supplementary Scheme S1). Therefore, the $\mathrm{p} K_{\mathrm{a}}$ values alone do not allow us to determine the correct tautomer form because all tautomers have the same number of $\mathrm{H}$ atoms and the same molecular charge (Haranczyk et al., 2008). When considering conventional refinement, as shown in Supplementary Tables S4 and S5, while the large number of possible tautomers did allow conventional refinement to yield XModeScores and ZDD scores with comparable standard deviations, the conventional refinement was unable to determine the correct tautomer.

\section{Conclusions}

With the calculations performed to date involving protomer/ tautomer-state determination, XModeScore has shown itself to be versatile and robust. Further, while the method could be used with either QM-based refinement or conventional refinement, the significance of the QM-based results clearly appears to be noticeably higher than that observed in conventional refinement even when advanced types of ligand restraints (e.g. Mogul CIF) are employed. Another related area of interest is in the exploration of heavy-atom flip-state ambiguity often observed in macromolecular X-ray crystallography. X-ray studies of protein-ligand complexes reliably reveal only the configuration of heavy atoms of the structure, with the caveat that elements with similar atomic numbers, such as $\mathrm{N}$ and $\mathrm{O}$, are often indistinguishable at modest resolutions. This leads to ambiguous orientations of molecule fragments capable of flipping, such as imidazole rings, amide groups and so on. Serious challenges in assigning the correct ligand orientation/flipping in X-ray macromolecular crystallography have been well documented and recognized (Malde \& Mark, 2011). Often, the hypothetical flip state is chosen based upon its agreement with the hydrogen-bond network and van der Waals contacts with the residue in question (Word et al., 1999). Not only does our method offer an entirely new X-ray data-driven approach for selecting flip states, but broadly speaking, any docking/placement of a ligand within the 'blob' of electron density can be addressed using our method. Further studies of this phenomenon will be explored in subsequent work.

\section{Acknowledgements}

We thank the National Institutes of Health (NIH) through SBIR \#R42GM079899 for funding the research and development effort.

\section{References}

Adams, P. D. et al. (2010). Acta Cryst. D66, 213-221.

Afonine, P. V., Mustyakimov, M., Grosse-Kunstleve, R. W., Moriarty, N. W., Langan, P. \& Adams, P. D. (2010). Acta Cryst. D66, 11531163.

Ahmed, H. U., Blakeley, M. P., Cianci, M., Cruickshank, D. W. J., Hubbard, J. A. \& Helliwell, J. R. (2007). Acta Cryst. D63, 906-922.

Bacon, G. E. (1975). Neutron Diffraction, 3rd ed. Oxford University Press.

Biffinger, J. C., Kim, H. W. \& DiMagno, S. G. (2004). Chembiochem, 5, 622-627.

Blum, M.-M., Mustyakimov, M., Ruterjans, H., Kehe, K., Schoenborn, B. P., Langan, P. \& Chen, J. C.-H. (2009). Proc. Natl Acad. Sci. USA, 106, 713-718.

Borbulevych, O., Merz, K. M. Jr \& Westerhoff, L. M. (2011). Acta Cryst. A67, C593.

Borbulevych, O. Y., Plumley, J. A., Martin, R. I., Merz, K. M. \& Westerhoff, L. M. (2014). Acta Cryst. D70, 1233-1247.

Coates, L., Erskine, P. T., Mall, S., Williams, P. A., Gill, R. S., Wood, S. P. \& Cooper, J. B. (2003). Acta Cryst. D59, 978-981.

Coates, L., Tuan, H.-F., Tomanicek, S., Kovalevsky, A., Mustyakimov, M., Erskine, P. \& Cooper, J. (2008). J. Am. Chem. Soc. 130, 72357237.

Cooper, J. B. (2002). Curr. Drug Targets, 3, 155-173.

Davies, D. R. (1990). Annu. Rev. Biophys. Biophys. Chem. 19, 189-215.

Dewar, M. J. S., Zoebisch, E. G., Healy, E. F. \& Stewart, J. J. P. (1985). J. Am. Chem. Soc. 107, 3902-3909.

Diller, D. J., Humblet, C., Zhang, X. \& Westerhoff, L. M. (2010). Proteins, 78, 2329-2337.

Dixon, S. L. \& Merz, K. M. (1996). J. Chem. Phys. 104, 6643-6649.

Dixon, S. L. \& Merz, K. M. (1997). J. Chem. Phys. 107, 879-893.

Duarte, H. A., Carvalho, S., Paniago, E. B. \& Simas, A. M. (1999). J. Pharm. Sci. 88, 111-120.

Eder, J., Hommel, U., Cumin, F., Martoglio, B. \& Gerhartz, B. (2007). Curr. Pharm. Des. 13, 271-285.

Fisher, S. Z., Aggarwal, M., Kovalevsky, A. Y., Silverman, D. N. \& McKenna, R. (2012). J. Am. Chem. Soc. 134, 14726-14729. 
Fu, Z., Li, X. \& Merz, K. M. (2012). J. Chem. Theory Comput. 8, 1436-1448.

Gibbons, J. D. \& Chakraborti, S. (2010). Nonparametric Statistical Inference, 5th ed. Boca Raton: Chapman \& Hall/CRC.

Gómez-Nuñez, M., Haro, K. J., Dao, T., Chau, D., Won, A., EscobarAlvarez, S., Zakhaleva, V., Korontsvit, T., Gin, D. Y. \& Scheinberg, D. A. (2008). PLoS One, 3, e3938.

Haranczyk, M., Puzyn, T. \& Sadowski, P. (2008). QSAR Comb. Sci. 27, 826-833.

Harding, M. M. (1999). Acta Cryst. D55, 1432-1443.

Howard, E. I., Sanishvili, R., Cachau, R. E., Mitschler, A., Chevrier, B., Barth, P., Lamour, V., Van Zandt, M., Sibley, E., Bon, C., Moras, D., Schneider, T. R., Joachimiak, A. \& Podjarny, A. (2004). Proteins, 55, 792-804.

Katz, A. K., Li, X., Carrell, H. L., Hanson, B. L., Langan, P., Coates, L., Schoenborn, B. P., Glusker, J. P. \& Bunick, G. J. (2006). Proc. Natl Acad. Sci. USA, 103, 8342-8347.

Kleywegt, G. J. (2007). Acta Cryst. D63, 94-100.

Kleywegt, G. J., Henrick, K., Dodson, E. J. \& van Aalten, D. M. F. (2003). Structure, 11, 1051-1059.

Krieger, E., Dunbrack, R. Jr, Hooft, R. W. W. \& Krieger, B. (2012). Methods Mol. Biol. 819, 405-421.

Krishnamurthy, V. M., Kaufman, G. K., Urbach, A. R., Gitlin, I., Gudiksen, K. L., Weibel, D. B. \& Whitesides, G. M. (2008). Chem. Rev. 108, 946-1051.

Labute, P. (2009). Proteins, 75, 187-205.

Lesburg, C. A., Huang, C., Christianson, D. W. \& Fierke, C. A. (1997). Biochemistry, 36, 15780-15791.

Malde, A. K. \& Mark, A. E. (2011). J. Comput. Aided Mol. Des. 25, $1-12$.

Martin, Y. C. (2009). J. Comput. Aided Mol. Des. 23, 693-704.

Martin, Y. C. (2010). Quantitative Drug Design: A Critical Introduction, 2nd ed. Boca Raton: CRC Press.

Merz, K. M. \& Banci, L. (1997). J. Am. Chem. Soc. 119, 863-871.

Moldow, B., Sander, B., Larsen, M. \& Lund-Andersen, H. (1999). Invest. Ophthalmol. Vis. Sci. 40, 1770-1775.

Moriarty, N. W., Grosse-Kunstleve, R. W. \& Adams, P. D. (2009). Acta Cryst. D65, 1074-1080.

Müller, K., Faeh, C. \& Diederich, F. (2007). Science, 317, 18811886.

Oksanen, E., Blakeley, M. P., El-Hajji, M., Ryde, U. \& BudayovaSpano, M. (2014). PLoS One, 9, e86651.

Piepenbrink, K. H., Borbulevych, O. Y., Sommese, R. F., Clemens, J., Armstrong, K. M., Desmond, C., Do, P. \& Baker, B. M. (2009). Biochem. J. 423, 353-361.

Pospisil, P., Ballmer, P., Scapozza, L. \& Folkers, G. (2003). J. Recept. Signal Transduct. Res. 23, 361-371.
Raczyńska, E. D., Kosińska, W., Ośmiałowski, B. \& Gawinecki, R. (2005). Chem. Rev. 105, 3561-3612.

Raha, K., Peters, M. B., Wang, B., Yu, N., Wollacott, A. M., Westerhoff, L. M. \& Merz, K. M. (2007). Drug Discov. Today, 12, 725-731.

Raha, K., van der Vaart, A. J., Riley, K. E., Peters, M. B., Westerhoff, L. M., Kim, H. \& Merz, K. M. (2005). J. Am. Chem. Soc. 127, 65836594.

Read, R. J. (1986). Acta Cryst. A42, 140-149.

Řezáč, J., Fanfrlík, J., Salahub, D. \& Hobza, P. (2009). J. Chem. Theory Comput. 5, 1749-1760.

Rupp, B. (2009). Biomolecular Crystallography: Principles, Practice, and Application to Structural Biology. New York: Garland Science.

Sampson, R. A. (1904). Mem. R. Astron. Soc. 54, 143-161.

Shannon, C. E. (1949). Proc. Inst. Radio Eng. 37, 10-21.

Shu, F., Ramakrishnan, V. \& Schoenborn, B. P. (2000). Proc. Natl Acad. Sci. USA, 97, 3872-3877.

Šidák, Z. K. (1967). J. Am. Stat. Assoc. 62, 626-633.

Simic, M. G. \& Jovanovic, S. V. (1989). J. Am. Chem. Soc. 111, 57785782.

Sippel, K. H., Robbins, A. H., Domsic, J., Genis, C., AgbandjeMcKenna, M. \& McKenna, R. (2009). Acta Cryst. F65, 992-995.

Stewart, J. J. P. (2009). J. Mol. Model. 15, 765-805.

Tickle, I. J. (2012). Acta Cryst. D68, 454-467.

Tomanicek, S. J., Standaert, R. F., Weiss, K. L., Ostermann, A., Schrader, T. E., Ng, J. D. \& Coates, L. (2013). J. Biol. Chem. 288, 4715-4722.

United States Pharmacopeia (1995). United States Pharmacopeia, 15th ed., Vol. 1, p. 659. Rockville: The United States Pharmacopeial Convention Inc.

Vaart, A. van der \& Merz, K. M. (1999). J. Phys. Chem. A, 103, 33213329.

Varga, L. I., Ako-Agugua, N., Colasante, J., Hertweck, L., Houser, T., Smith, J., Watty, A. A., Nagar, S. \& Raffa, R. B. (2009). J. Clin. Pharm. Ther. 34, 493-505.

Warr, W. (2010). J. Comput. Aided Mol. Des. 24, 497-520.

Wlodawer, A., Minor, W., Dauter, Z. \& Jaskolski, M. (2008). FEBS J. 275, $1-21$.

Word, J. M., Lovell, S. C., Richardson, J. S. \& Richardson, D. C. (1999). J. Mol. Biol. 285, 1735-1747.

Yu, N., Hayik, S. A., Wang, B., Liao, N., Reynolds, C. H. \& Merz, K. M. (2006). J. Chem. Theory Comput. 2, 1057-1069.

Yu, N., Yennawar, H. P. \& Merz, K. M. (2005). Acta Cryst. D61, 322-332.

Yuriev, E. \& Ramsland, P. A. (2013). J. Mol. Recognit. 26, 215-239.

Zhang, X., Gibbs, A. C., Reynolds, C. H., Peters, M. B. \& Westerhoff, L. M. (2010). J. Chem. Inf. Model. 50, 651-661. 